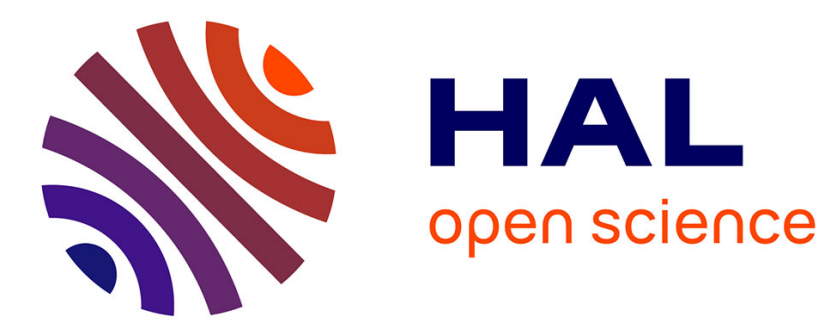

\title{
Fault diagnosis for a class of descriptor linear parameter varying systems
}

\author{
Carlos Manuel Astorga Zaragoza, Didier Theilliol, Jean-Christophe Ponsart, \\ Mickael Rodrigues
}

\section{- To cite this version:}

Carlos Manuel Astorga Zaragoza, Didier Theilliol, Jean-Christophe Ponsart, Mickael Rodrigues. Fault diagnosis for a class of descriptor linear parameter varying systems. International Journal of Adaptive Control and Signal Processing, 2012, 26 (3), pp.208-223. 10.1002/acs.1259 . hal-00605392

\section{HAL Id: hal-00605392 https://hal.science/hal-00605392}

Submitted on 1 Jul 2011

HAL is a multi-disciplinary open access archive for the deposit and dissemination of scientific research documents, whether they are published or not. The documents may come from teaching and research institutions in France or abroad, or from public or private research centers.
L'archive ouverte pluridisciplinaire HAL, est destinée au dépôt et à la diffusion de documents scientifiques de niveau recherche, publiés ou non, émanant des établissements d'enseignement et de recherche français ou étrangers, des laboratoires publics ou privés. 


\title{
Fault diagnosis for a class of Descriptor Linear Parameter Varying systems
}

\author{
C. M. Astorga-Zaragoza ${ }^{1}$, D. Theilliol ${ }^{2, *}$, J. C. Ponsart ${ }^{2}$ and M. Rodrigues ${ }^{3}$ \\ ${ }^{1}$ Centro Nacional de Investigación y Desarrollo Tecnológico, CENIDET, Internado Palmira s/n, \\ Col. Palmira, C.P. 62490, Cuernavaca, Mor., Mexico \\ ${ }^{2}$ Centre de Recherche en Automatique de Nancy, CRAN CNRS UMR 7039, \\ Nancy-Université, France \\ ${ }^{3}$ Laboratoire d'Automatique et de Génie des Procédés (LAGEP), CNRS UMR 500\%, \\ Université Lyon 1, France
}

\begin{abstract}
SUMMARY
In this paper, a model-based fault estimation method for a particular class of discrete-time Descriptor Linear Parameter Varying (D-LPV) systems is developed. The main contribution of this work consists in the design of an observer that performs simultaneously both, the states estimation and the fault magnitude vectors, considered as unknown inputs. The conditions for the existence of such observer are given. Such conditions guarantee the observer stability and they are proved through a Lyapunov analysis combined with a Linear Matrix Inequalities (LMI) formulation. The fault estimation scheme is evaluated through numerical simulations. Copyright (c) 2002 John Wiley \& Sons, Ltd.
\end{abstract}

KEY WORDS: fault estimation; observers; LPV systems; descriptor systems

\section{INTRODUCTION}

Descriptor systems (also known as singular systems) are a special class of systems that include ordinary algebraic equations, (differential) state-space equations, combinations of algebraic and differential equations and noncausal systems [1]. From the point of view of process modeling, descriptor systems can be considered as a generalization of state-space linear systems where algebraic relations resulting from the interaction of the process dynamics exist. Several works dealing with linear and nonlinear descriptor systems exist in literature (see for instance [2] for linear systems and [4] for the nonlinear case). Some recent works dealing with descriptor systems are $[5,6,7]$ where different observer approaches are used for this kind of systems. Different fault diagnosis techniques for descriptor systems are recently synthesized in $[8,9]$.

On the other hand, Linear Parameter Varying (LPV) systems (which can be considered as a particular case of Linear Time Varying systems LTV) can be used to approximate nonlinear systems, hence systematic and generic available theoretical results for LPV systems

*Correspondence to: didier.theilliol@cran.uhp-nancy.fr 
can be applied to derive nonlinear control laws for nonlinear systems. For instance, in [10] the authors use the LPV approach to model and control diesel engines. In [11] a method for designing structured fault diagnosis filters (with guaranteed $H_{\infty}$ nuisance attenuation and fault transmission $H_{-}$gain) for polytopic LPV systems is developed and simulated for a secondary circuit of a nuclear power plant. An application of fault detection and isolation to a Boeing $747-100 / 200$ aircraft is presented in [12].

In recent years, fault detection and isolation for LPV systems has been investigated for several authors. The case of robust fault detection and isolation of multiple simultaneous faults for affine LPV systems affected by disturbances is presented in [13], where the authors design a residual generator filter that maximizes the DC-transmission gain from a specific fault to the residual while attenuating the effect caused by the nuisances. The authors in [14] present an $H_{\infty}$ approach for fault detection and isolation (and estimation), where a linear fractional transformation (LFT) representation of the LPV system is adopted. In $[15,16]$, failure detection for LPV systems is investigated by using a geometric approach, where a procedure was derived to obtain the detection filter gain via the construction of a suitable family of invariant subspaces. Parameter-dependent observers with pole assignment are used to synthesize the residual generators for LPV systems in [17, 18, 19]. A robust state-based approach to estimate faults in uncertain LPV systems can be found in [20].

Although the idea of merging descriptor and LPV systems is not new (see for instance [21]), there are very few works dealing with observer and controller synthesis, stabilization, fault diagnosis and other topics on automatic control for this kind of systems. Some recent papers on this topic dealing with the problem of control and stabilization are [22, 23], whereas the authors in [24] addresses the problem of observer-based controllers.

The aim of this work is to develop a fault estimation method for discrete-time descriptor linear parameter varying systems (in the sequel referred as D-LPV systems), described by a polytopic system representation. In order to achieve this objective, an observer for D-LPV systems is synthesized. The observer is based on the observer proposed in [25] where a method to design full-order observers for LTI descriptor systems is considered. The existence conditions of the observer are given. Such conditions guarantee the observer convergence proved through a combined method based on the original approach proposed by [25] and a Lyapunov analysis.

By taking into account some simple matrix manipulations, this observer can estimate both, the states and the unknown inputs, simultaneously. In order to achieve a robust fault detection and identification, the unknown input estimation feature is used to build a fault diagnosis scheme based on a bank of observers. The effectiveness and performance of the proposed scheme are illustrated through a numerical example.

\section{PROBLEM FORMULATION}

Consider the following discrete-time polytopic D-LPV system where the terms $k$ and $k+1$ are the simplified form to write $k T_{s}$ and $k T_{s}+T_{s}$ respectively, and $T_{s}$ is the sampling time:

$$
\begin{aligned}
\bar{E} \tilde{x}(k+1) & =\sum_{i=1}^{M} \varepsilon_{i}(\rho(k))\left(\bar{A}_{i} \tilde{x}(k)+\bar{B}_{i} u(k)\right)+\bar{F} v(k) \\
y(k) & =\bar{C} \tilde{x}(k)
\end{aligned}
$$


where $\tilde{x}(k) \in \mathbb{R}^{n}, u(k) \in \mathbb{R}^{q}, y(k) \in \mathbb{R}^{p}, v(k) \in \mathbb{R}^{d}$ are the state, the measured input, the measured output and the unmeasured input vectors which can be classically considered as fault vectors, respectively, $\bar{E} \in \mathbb{R}^{m \times n}, \bar{A}_{i} \in \mathbb{R}^{m \times n}, \bar{B}_{i} \in \mathbb{R}^{n \times q}, \bar{C} \in \mathbb{R}^{p \times n}, \bar{F} \in \mathbb{R}^{n \times d}$, are constant matrices, $\rho(k)$ is a time-varying bounded parameter vector assumed to be measured on-line and $M$ is the total number of weighting functions $\varepsilon_{i}(\rho(k))$ defined as

$$
\sum_{i=1}^{M} \varepsilon_{i}(\rho(k))=1, \quad \varepsilon_{i}(\rho(k)) \geq 0
$$

Classicaly, the term polytopic comes from the fact that the vector $\varepsilon(\rho(k))$ evolves over the convex set:

$$
\Omega=\left\{\begin{array}{c}
\text { col } \\
i
\end{array}: \sum_{i=1}^{M} \varepsilon_{i}(\rho(k))=1, \varepsilon_{i}(\rho(k)) \geq 0\right\}
$$

The following assumptions are considered ([25]):

(A1) $\operatorname{rank} \bar{E}=r<n$.

(A2) $\operatorname{rank}\left(\begin{array}{c}\bar{E} \\ \bar{C}\end{array}\right)=n$.

Assumption A1 simply states that $\bar{E}$ is a singular matrix. Assumption A2, guarantees the existence of a nonsingular matrix $\bar{\Gamma}$ :

$$
\bar{\Gamma}=\left(\begin{array}{cc}
\bar{\alpha} & \bar{\beta} \\
\bar{\gamma} & \bar{\xi}
\end{array}\right)
$$

such that

$$
\begin{gathered}
\bar{\alpha} \bar{E}+\bar{\beta} \bar{C}=I_{n} \\
\bar{\gamma} \bar{E}+\bar{\xi} \bar{C}=0
\end{gathered}
$$

where $\bar{\alpha}, \bar{\beta}, \bar{\gamma}$ and $\bar{\xi}$ are constant matrices of appropriate dimensions which can be found by the singular value decomposition of $\left(\begin{array}{c}\bar{E} \\ \bar{C}\end{array}\right)$.

The problem is to synthesize an observer for system (1) such that the estimation error $\tilde{e}(k)=\tilde{x}(k)-\hat{\tilde{x}}(k)$ converges to zero as $k \rightarrow \infty$.

In this paper bias are considered constant faults, called bias, are considered, i.e.

(A3) $v(k)=v(k-1)$.

This assumption may be considered as restrictive, however in many practical situations, once a fault appears, it remains constant during a significant time period that is long enough to estimate the fault. In this way, the system (1) can be rewritten in the following form:

$$
\begin{aligned}
E x(k+1) & =\sum_{i=1}^{M} \varepsilon_{i}(\rho(k))\left(A_{i} x(k)+B_{i} u(k)\right) \\
y(k) & =C x(k)
\end{aligned}
$$

Copyright (c) 2002 John Wiley \& Sons, Ltd. Prepared using acsauth.cls 
where

$$
\begin{gathered}
E=\left(\begin{array}{cc}
\bar{E} & -\bar{F} \\
0_{d \times n} & I_{d}
\end{array}\right) \\
A_{i}=\left(\begin{array}{cc}
\bar{A}_{i} & 0_{m \times d} \\
0_{d \times n} & I_{d}
\end{array}\right) \\
B_{i}=\left(\begin{array}{c}
\bar{B}_{i} \\
0_{d \times q}
\end{array}\right) \\
C=\left(\begin{array}{ll}
\bar{C} & 0_{p \times d}
\end{array}\right)
\end{gathered}
$$

Following the idea presented by Darouach [7] for state and unknown input estimation, the expanded state vector is formed by the usual state variables and the faults at the precedent time instant

$$
x(k)=\left(\begin{array}{c}
\tilde{x}(k) \\
v(k-1)
\end{array}\right) \in \mathbb{R}^{(m+d) \times(n+d)}
$$

In this way, the faults can be estimated simultaneously with the state variables. The problem becomes to design an adequate observer for the D-LPV system (5).

Assumption A2 becomes

$$
\text { (A4) } \operatorname{rank}\left(\begin{array}{c}
E \\
C
\end{array}\right)=n+d \text {. }
$$

Considering Assumption A4, it exists a nonsingular matrix $\Gamma$ :

$$
\Gamma=\left(\begin{array}{cc}
\alpha & \beta \\
\gamma & \xi
\end{array}\right)
$$

such that

$$
\begin{gathered}
\alpha E+\beta C=I_{n+d} \\
\gamma E+\xi C=0
\end{gathered}
$$

where $\alpha, \beta, \gamma$ and $\xi$ are constant matrices of appropriate dimensions which can be found by the singular value decomposition of $\left(\begin{array}{c}E \\ C\end{array}\right)$.

The following additional assumption is considered:

(A5) The pair $\left(\alpha A_{i},\left(\begin{array}{c}\gamma A_{i} \\ C\end{array}\right)\right)$ is detectable.

As proposed in [25], if Assumptions A4 and A5 holds, an observer for the system (5) should have the following form:

$$
\begin{aligned}
z(k+1) & =\sum_{i=1}^{M} \varepsilon_{i}(\rho(k))\left[N_{i} z(k)+L_{1 i} y(k)+G_{i} u(k)+L_{2 i} y(k)\right] \\
\hat{x}(k) & =z(k)+\beta y(k)+K \xi y(k)
\end{aligned}
$$


where $z(k) \in \mathbb{R}^{n+d}$ is the observer state vector; $N_{i}, L_{1 i}, L_{2 i}, G_{i}$ and $K, i=1, \ldots, M$ are constant matrices of appropriate dimensions.

In the next section, sufficient conditions are given, such that system (13) is an observer for system (5).

\section{THE OBSERVER SYNTHESIS}

For the sake of simplicity, the following notation is used:

$$
\Omega\left(\rho_{k}\right)=\sum_{i=1}^{M} \varepsilon_{i}(\rho(k)) \Omega_{i} \quad i=1, \ldots, M
$$

Thus, system (13) can be rewritten in the simplified form:

$$
\begin{aligned}
z(k+1) & =N\left(\rho_{k}\right) z(k)+L_{1}\left(\rho_{k}\right) y(k)+G\left(\rho_{k}\right) u(k)+L_{2}\left(\rho_{k}\right) y(k) \\
\hat{x}(k) & =z(k)+\beta y(k)+K \xi y(k)
\end{aligned}
$$

Let the observer error $e(k) \in \mathbb{R}^{n+d}$ be defined as:

$$
e(k)=x(k)-\hat{x}(k)
$$

The difference equation of the error is

$$
\begin{aligned}
e(k+1) & =\left[(\alpha+K \gamma) A\left(\rho_{k}\right)-N\left(\rho_{k}\right)(\alpha+K \gamma) E-L_{1}\left(\rho_{k}\right) C-L_{2}\left(\rho_{k}\right) C\right] x(k) \\
& +\left[(\alpha+K \gamma) B\left(\rho_{k}\right)-G\left(\rho_{k}\right)\right] u(k)+N\left(\rho_{k}\right) \underbrace{[(\alpha+K \gamma) E x(k)-z(k)]}_{e(k)}
\end{aligned}
$$

If the following conditions are fulfilled

$$
\begin{gathered}
G\left(\rho_{k}\right)=(\alpha+K \gamma) B\left(\rho_{k}\right) \\
L_{1}\left(\rho_{k}\right)=N\left(\rho_{k}\right)(\beta+K \xi)
\end{gathered}
$$

and

$$
N\left(\rho_{k}\right)=K \gamma A\left(\rho_{k}\right)+\alpha A\left(\rho_{k}\right)-L_{2}\left(\rho_{k}\right) C
$$

then (17) reduces to

$$
e(k+1)=N\left(\rho_{k}\right) e(k)
$$

Equation (21) can be written as:

$$
e(k+1)=\sum_{i=1}^{M} \varepsilon_{i}(\rho(k))\left(\tilde{A}_{i}+\tilde{K}_{i} \tilde{C}_{i}\right) e(k)
$$


where $\tilde{A}_{i}=\alpha A_{i}, \tilde{K}_{i}=\left(\begin{array}{ll}K & -L_{2 i}\end{array}\right)$ and $\tilde{C}_{i}=\left(\begin{array}{c}\gamma A_{i} \\ C\end{array}\right)$.

Equation (22) confirms the necessity of Assumption A5. The following subsection is devoted to demonstrated the stability and convergence of the error dynamics given in Equation (22).

\subsection{Observer stability}

Theorem 3.1. Considering Assumptions A4-A5, the system (15) is a stable observer for the system (5) if there exist symmetric positive definite matrices $H_{i}$, nonsingular matrices $J_{i}$, and matrices $L_{2 i}$, and $K$ such that

$$
\left(\begin{array}{ccc}
J_{i}^{T}+J_{i} & N_{i}^{T} & J_{i}^{T} \\
N_{i} & H_{j} & 0 \\
J_{i} & 0 & H_{i}^{-1}
\end{array}\right)>0
$$

$\forall i=1, \ldots, M, j=1, \ldots, M$, where $N_{i}=K \gamma A_{i} G_{i}+\alpha A_{i} G_{i}-L_{2 i} C G_{i}$.

Proof:

Consider the following Parameter Dependent Lyapunov Function (PDLF)

$$
V(e(k), \xi(\rho(k)))=e^{T}(k) P\left(\rho_{k}\right) e(k)
$$

where according to notation (14):

$$
P\left(\rho_{k}\right)=\sum_{i=1}^{M} \varepsilon_{i}(\rho(k)) P_{i}
$$

$P_{i}$ are symmetric positive definite constant matrices. According to the Lyapunov stability theorem, this PDLF must satisfy:

$$
\begin{aligned}
\Delta V(k) & =V(k+1)-V(k) \\
& =e^{T}(k)\left(N^{T}\left(\rho_{k}\right) P\left(\rho_{k+1}\right) N\left(\rho_{k}\right)-P\left(\rho_{k}\right)\right) e(k) \leq 0
\end{aligned}
$$

It can be seen that the function $\Delta V(k)$ is negative definite if

$$
N^{T}\left(\rho_{k}\right) P\left(\rho_{k+1}\right) N\left(\rho_{k}\right)-P\left(\rho_{k}\right)<0
$$

The following notation is used for $P\left(\rho_{k+1}\right)$ :

$$
P\left(\rho_{k+1}\right)=\sum_{i=1}^{M} \varepsilon_{i}(\rho(k+1)) P_{i}=\sum_{j=1}^{M} \varepsilon_{j}(\rho(k)) P_{j}
$$

By substituting (25) and (28) in (27), it can be deduced that the negative definiteness of (27) is guaranteed if [26]:

$$
N_{i}^{T} P_{j} N_{i}-P_{i}<0, \forall i=1, \ldots, M, j=1, \ldots, M .
$$

The authors in [26] have demonstrated that poly-quadratically stability of the system $e(k+1)=N\left(\rho_{k}\right) e(k)$ (which ensures the stability of the observer) is guaranteed if and only if 
there exist symmetric positive definite matrices $H_{i}$, and matrices (of appropriate dimensions) $\Psi_{i}, i=1, \ldots, M$, such that

$$
\left(\begin{array}{cc}
\Psi_{i}+\Psi_{i}^{T}-H_{i} & \Psi_{i}^{T} N_{i}^{T} \\
N_{i} \Psi_{i} & H_{j}
\end{array}\right)>0
$$

$\forall i=1, \ldots, M, j=1, \ldots, M$, with

$$
P\left(\rho_{k}\right)=\sum_{i=1}^{M} \varepsilon_{i}(\rho(k)) H_{i}^{-1}
$$

It is worth to note from equations $(25),(28)$ and (31) that $H_{i}=P_{i}^{-1}$ and $H_{j}=P_{j}^{-1}$. In this way, the feasibility problem of inequalities (29) can be replaced for the feasibility problem of inequality (30). If so, then (26) is a negative definite and decreasing sequence.

Replacing $N_{i}$ from (20) into (30):

$$
\left(\begin{array}{cc}
\Psi_{i}+\Psi_{i}^{T}-H_{i} & (\star)^{T} \\
K \gamma A_{i} \Psi_{i}+\alpha A_{i} \Psi_{i}-L_{2 i} C \Psi_{i} & H_{j}
\end{array}\right)>0
$$

$\forall i=1, \ldots, M, j=1, \ldots, M$. It can be seen that the main difficulty to solve the inequality (32) is the bilinear term $K \gamma A_{i} \Psi_{i}$, where $K$ and $\Psi_{i}$ are unknown matrices.

Assuming that $\Psi_{i}$ is nonsingular, the positive definiteness of (30) is equivalent to the positive definiteness of the following congruent matrix

$$
\left(\begin{array}{cc}
\left(\Psi_{i}^{-1}\right)^{T} & 0 \\
0 & I
\end{array}\right)\left(\begin{array}{cc}
\Psi_{i}+\Psi_{i}^{T}-H_{i} & \Psi_{i}^{T} N_{i}^{T} \\
N_{i} \Psi_{i} & H_{j}
\end{array}\right)\left(\begin{array}{cc}
\Psi_{i}^{-1} & 0 \\
0 & I
\end{array}\right)>0
$$

or

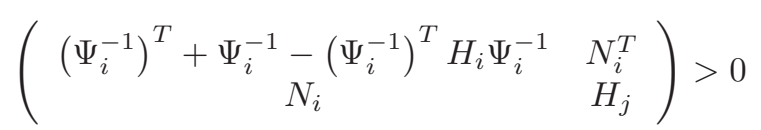

Equation (34) is the Schur complement of the following positive definite matrix

$$
\left(\begin{array}{ccc}
J_{i}^{T}+J_{i} & N_{i}^{T} & J_{i}^{T} \\
N_{i} & H_{j} & 0 \\
J_{i} & 0 & H_{i}^{-1}
\end{array}\right)>0
$$

with $J_{i}=\Psi_{i}^{-1}$. This completes the proof.

The inequality (23), implies that $H_{j} H_{i}^{-1}=I$ when $i=j$, or simply

$$
H_{i} P_{i}=I
$$

(where $P_{i}=H_{i}^{-1}$ ). Thus, inequality (23) can be written as:

$$
\left(\begin{array}{ccc}
J_{i}^{T}+J_{i} & N_{i}^{T} & J_{i}^{T} \\
N_{i} & H_{j} & 0 \\
J_{i} & 0 & P_{i}
\end{array}\right)>0, H_{i} P_{i}=I .
$$


Among various methods to solve LMI problem under a LME constraint, the cone complementary technique has been considered. The following facts are used to give a solution procedure to solve the inequality (37):

Fact 3.1. The feasibility of the LMI

$$
\left(\begin{array}{cc}
H_{i} & I \\
I & P_{i}
\end{array}\right) \geq 0, H_{i}>0 ; P_{i}>0 i=1, \ldots, M
$$

implies that $\operatorname{Tr}\left(H_{i} P_{i}\right) \geq n$, where the equality $\operatorname{Tr}\left(H_{i} P_{i}\right)=n$ holds if and only if $H_{i} P_{i}=I$ (see [27]).

The following matrices are introduced

$$
\begin{aligned}
\mathbb{H} & =\operatorname{diag}\left(H_{1}, \ldots, H_{M}\right) \\
\mathbb{P} & =\operatorname{diag}\left(P_{1}, \ldots, P_{M}\right)
\end{aligned}
$$

By taking into account Fact 3.1, it is easy to deduce the following:

Fact 3.2. The feasibility of the LMI

$$
\left(\begin{array}{cc}
\mathbb{H} & I \\
I & \mathbb{P}
\end{array}\right) \geq 0, \mathbb{H}>0 ; \mathbb{P}>0
$$

implies that $\operatorname{Tr}(\mathbb{H} \mathbb{P}) \geq n M$, where the equality $\operatorname{Tr}(\mathbb{H P})=n M$ holds if and only if $\mathbb{H} \mathbb{P}=I$

In this way, the problem of finding a solution of (37) is equivalent to solve the following minimization problem:

$$
\min \operatorname{Tr}(\mathbb{H} \mathbb{P}) \text { subject to }\left\{\begin{array}{ccc}
\left(\begin{array}{ccc}
J_{i}^{T}+J_{i} & N_{i}^{T} & J_{i}^{T} \\
N_{i} & H_{j} & 0 \\
J_{i} & 0 & P_{i}
\end{array}\right)>0 \\
& \left(\begin{array}{cc}
\mathbb{H} & I \\
I & \mathbb{P}
\end{array}\right)>0 \\
& & \mathbb{H}>0 \\
& & \mathbb{P}>0
\end{array}\right.
$$

$\forall i=1 \ldots, M$ and $\forall j=1 \ldots, M$. The bilinear objective problem: $\min \operatorname{Tr}(\mathbb{H} \mathbb{P})$, in $(40)$ is solved by using the cone complementary algorithm proposed in [28].

The following notation is introduced: $H^{[\mathrm{k}]}$ is used to denote the $\mathrm{k}$-th element of the matrix sequence $H^{[0]}, H^{[1]}, \ldots, H^{\left[\mathrm{k}_{o p t}\right]}$. 
The cone complementary algorithm propose to transform the bilinear objective problem given in (40) by a linear objective problem of the form

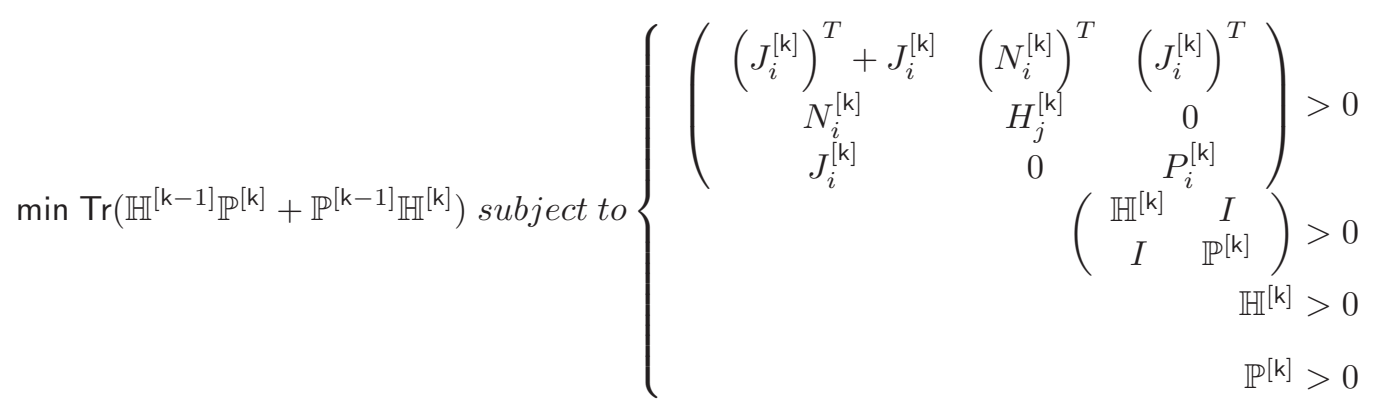

for $\mathrm{k}=1, \ldots, \mathrm{k}_{\text {opt }}$, and to find recursively the values of $\mathbb{H}=\mathbb{H}^{\left[\mathrm{k}_{\text {opt }}\right]}, \mathbb{P}=\mathbb{P}^{\left[\mathrm{k}_{o p t}\right]}, J_{i}=J_{i}^{\left[\mathrm{k}_{o p t}\right]}$, $N_{i}=N_{i}^{\left[\mathrm{k}_{o p t}\right]}$, where $\mathrm{k}_{\text {opt }}$ represents the iteration number where the sequence converges to $\min \operatorname{Tr}\left(\mathbb{H}^{[\mathrm{k}-1]} \mathbb{P}^{[\mathrm{k}]}+\mathbb{P}^{[\mathrm{k}-1]} \mathbb{H}^{[\mathrm{k}]}\right)=2 n M$ or equivalently $\min \operatorname{Tr}(\mathbb{H} \mathbb{P})=n M$, in other words $\min \operatorname{Tr}\left(H_{i}^{[\mathrm{k}-1]} P_{i}^{[\mathrm{k}]}+P_{i}^{[\mathrm{k}-1]} H_{i}^{[\mathrm{k}]}\right)=2 n$ or equivalently $\min \operatorname{Tr}\left(H_{i} P_{i}\right)=n$.

A small enough value of a constant stopping criterion $\varepsilon>0$ should be considered with the purpose of reduce the convergence time of the algorithm, i.e., once the value of $\operatorname{Tr}\left(\mathbb{H}^{[\mathrm{k}]} \mathbb{P}^{[\mathrm{k}]}\right)<n M+\varepsilon$, the algorithm stops. As indicated [28], each step of the algorithm has a simple LMI problem. The recursive sequence is bounded and decreases if and only if $\mathbb{H}^{[\mathrm{k}]} \mathbb{P}^{[\mathrm{k}]}$ is at the optimum. For more details concerning the used of such method, the authors recommend to read carefully [28] and associated papers.

The initial matrices $\mathbb{H}^{[0]}$ and $\mathbb{P}^{[0]}$, are obtained by computing an initial feasible point of the simple LMIs

$$
\begin{aligned}
& \left(\begin{array}{ccc}
\left(J_{i}^{[0]}\right)^{T}+J_{i}^{[0]} & \left(N_{i}^{[0]}\right)^{T} & \left(J_{i}^{[0]}\right)^{T} \\
N_{i}^{[0]} & H_{j}^{[0]} & 0 \\
J_{i}^{[0]} & 0 & P_{i}^{[0]}
\end{array}\right)>0 \\
& \left(\begin{array}{cc}
\mathbb{H}^{[0]} & I \\
I & \mathbb{P}^{[0]}
\end{array}\right)>0 \\
& \mathbb{H}^{[0]}>0 \\
& \mathbb{P}^{[0]}>0
\end{aligned}
$$

$\forall i=1 \ldots, M$ and $\forall j=1 \ldots, M$.

\subsection{Observer gain design based on a pole placement}

The observer should generate an accurate state and unknown inputs estimations based on an appropriate gain design. Among various kind of LMI regions, such as disk, cone, subplane, a pole placement through a disk as proposed by [29] has been considered. In fact, any region included inside the disk $D(0,1)$ can be chosen. However, if pole placement design is not considered, then the algorithm used to solve (41) could compute any $H_{i}, J_{i}, L_{2 i}$ and $K$ satisfying the LMI (23), such that the matrices $N_{i}, i=1, \ldots, M$ have eigenvalues anywhere 
inside the unit circle, disregarding if they are near from the origin or near from the border of the unit circle. Then, in order to ensure an adequate pole placement of matrices $N_{i}, i=1, \ldots, n$, the following inequalities can be taken into account instead of the inequalities shown in (29):

$$
\left(N_{i}-\lambda_{i} I\right)^{T} P_{j}\left(N_{i}-\lambda_{i} I\right)-\delta_{i}^{2} P_{i}<0, \forall i=1, \ldots, M, j=1, \ldots, M
$$

By doing so, the poles of matrices $N_{i}$ are assigned in a disk $D\left(\lambda_{i}, \delta_{i}\right)$ defined by the radius $\delta_{i}>0$ and the real number $\lambda_{i} \in \mathbb{R}$ such that $\delta_{i} \leq 1-\left|\lambda_{i}\right|, \forall i=1, \ldots, M$. The parameters associated to a disk region are chosen from the nominal dynamic behavior of the system in order to stabilize and to guarantee an efficient state estimation.

The inequality shown in (43) can be written in the simplified form:

$$
\bar{N}_{i}^{T} P_{j} \bar{N}_{i}-\bar{P}_{i}<0, \forall i=1, \ldots, M, j=1, \ldots, M
$$

which have the same form of inequality (29) with $\bar{N}_{i}=\left(N_{i}-\lambda_{i} I\right)$ and $\bar{P}_{i}=\delta_{i}^{2} P_{i}$. According to Theorem 3.1, the feasibility of inequality (44) is equivalent to the feasibility of finding symmetric positive definite matrices $\bar{H}_{i}$, and matrices (of appropriate dimensions) $\Psi_{i}, i=1, \ldots, M$, such that

$$
\left(\begin{array}{cc}
\Psi_{i}+\Psi_{i}^{T}-\bar{H}_{i} & \Psi_{i}^{T} \bar{N}_{i}^{T} \\
\bar{N}_{i} \Psi_{i} & H_{j}
\end{array}\right)>0
$$

$\forall i=1, \ldots, M, j=1, \ldots, M$, with

$$
\bar{P}\left(\rho_{k}\right)=\sum_{i=1}^{M} \varepsilon_{i}(\rho(k)) \bar{H}_{i}^{-1}
$$

where $\bar{H}_{i}=\bar{P}_{i}^{-1}=\delta_{i}^{-2} P_{i}^{-1}=\delta_{i}^{-2} H_{i}$

Replacing $\bar{H}_{i}$ and $\bar{N}_{i}$ in (45), and by following an analogous procedure to that described in the proof of Theorem 3.1, the following corollary can be easily derived

Corollary 3.1. Considering Assumptions A4-A5, the system (15) is a stable observer for the system (5) if there exist symmetric positive definite matrices $H_{i}$ and nonsingular matrices $J_{i}$ such that

$$
\left(\begin{array}{ccc}
J_{i}^{T}+J_{i} & \left(N_{i}-\lambda_{i} I\right)^{T} & J_{i}^{T} \\
\left(N_{i}-\lambda_{i} I\right) & H_{j} & 0 \\
J_{i} & 0 & \delta_{i}^{2} H_{i}^{-1}
\end{array}\right)>0
$$

$\forall i=1, \ldots, M, j=1, \ldots, M$, where $N_{i}=K \gamma A_{i}+\alpha A_{i}-L_{2 i} C$ and $L_{2 i}$. The matrices $G\left(\rho_{k}\right)$, $L_{1}\left(\rho_{k}\right)$ and $N\left(\rho_{k}\right)$ are deduced from (18)-(20). The poles of $N_{i}$ are located in a disk $D\left(\lambda_{i}, \delta_{i}\right)$ defined by the radius $\delta_{i}>0$ and the real number $\lambda_{i} \in \mathbb{R}$ such that $\delta_{i} \leq 1-\left|\lambda_{i}\right|$.

Finally, the cone complementary algorithm transforms the problem of feasibility of the inequality shown in (47) in the following linear objective minimization problem: 


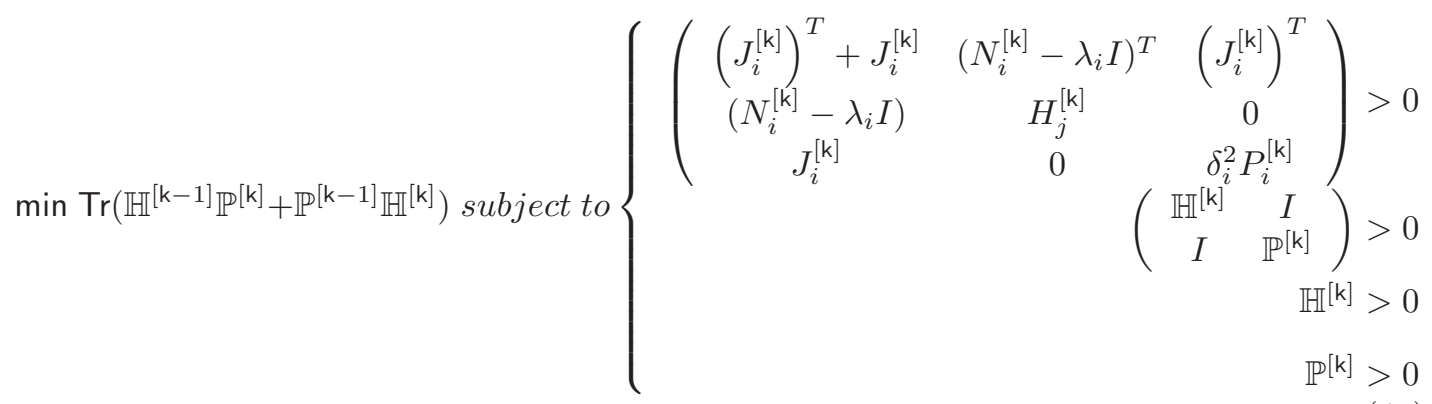

which is solved as described above. If (48) is feasible, then the poles of $N_{i}$ are located in a disk $D\left(\lambda_{i}, \delta_{i}\right)$ defined by the radius $\delta_{i}>0$ and the real number $\lambda_{i} \in \mathbb{R}$ such that $\delta_{i} \leq 1-\left|\lambda_{i}\right|$.

\subsection{Extended case}

In this subsection, the design of a full-order observer for descriptor systems with measurement noises and perturbations is presented.

Let us consider the signal $w(t)$ characterized as the output of the following linear discretetime system:

$$
\begin{aligned}
x_{w}(k+1) & =A_{w} x_{w}(k)+B_{w} u_{w}(k) \\
w(k) & =C_{w} x_{w}(k)
\end{aligned}
$$

where $x_{w}(k) \in \mathbb{R}^{w}, u_{w}(k) \in \mathbb{R}, w(k) \in \mathbb{R}$ are the state, the input and the output of the perturbation model. $A_{w}, B_{w}$ and $C_{w}$ are constant matrices of appropriate dimensions.

The signal $w(k)$ can represent either a perturbation signal, or a noise signal. The output $y_{m}(k)$ is given by

$$
y_{m}(k)=y(k)+w(k)=\left[\begin{array}{ll}
C & C_{w}
\end{array}\right]\left[\begin{array}{c}
x(k) \\
x_{w}(k)
\end{array}\right]
$$

The state-space models of systems (5) and (49) can be rewritten in the compact form:

$$
\begin{aligned}
\tilde{E} \dot{\bar{x}}(k+1) & =\tilde{A}\left(\rho_{k}\right) \bar{x}(k)+\tilde{B}\left(\rho_{k}\right) \bar{u}(k) \\
y_{m}(k) & =\tilde{C} \bar{x}(k)
\end{aligned}
$$

where

$$
\begin{gathered}
\tilde{E}=\left[\begin{array}{cc}
E & 0 \\
0 & I_{w}
\end{array}\right] \tilde{A}\left(\rho_{k}\right)=\left[\begin{array}{cc}
A\left(\rho_{k}\right) & 0 \\
0 & A_{w}
\end{array}\right] \tilde{B}\left(\rho_{k}\right)=\left[\begin{array}{cc}
B\left(\rho_{k}\right) & 0 \\
0 & B_{w}
\end{array}\right] \\
\tilde{C}=\left[\begin{array}{ll}
C & C_{w}
\end{array}\right] \bar{x}(k)=\left[\begin{array}{c}
x(k) \\
x_{w}(k)
\end{array}\right] \bar{u}(k)=\left[\begin{array}{c}
u(k) \\
u_{w}(k)
\end{array}\right]
\end{gathered}
$$

The procedure to design an observer for system (50) is equivalent to design an observer for system (5) extended to a classical robust residual generators method [3] . 


\section{ILLUSTRATIVE EXAMPLE}

Consider a descriptor LPV system of the form (1) in the presence of two structured faults with unknown fault magnitudes given by:

$$
\begin{gathered}
\bar{A}_{1}=\left(\begin{array}{cccc}
2.018 & 1.326 & -0.08691 & -0.1644 \\
2.277 & 2.018 & -0.1359 & -0.2908 \\
0.4259 & 0.1666 & 0.5714 & 0.1732 \\
0.6659 & 0.4259 & -0.4822 & -0.152
\end{array}\right) \bar{A}_{2}=\left(\begin{array}{cccc}
1 & 1.4 & -0.07 & -0.2 \\
1.5 & 2 & -0.15 & -0.3 \\
0.4 & 0.1 & 0.5 & 0.2 \\
0 & 0 & -0.5 & -0.2
\end{array}\right) \\
\bar{B}_{1}=\left(\begin{array}{c}
0.6047 \\
1.644 \\
-1.709 \\
-1.732
\end{array}\right) \bar{B}_{2}=\left(\begin{array}{c}
0.5 \\
1 \\
-1.5 \\
-1
\end{array}\right) \bar{C}=\left(\begin{array}{llll}
1 & 0 & 0 & 0 \\
0 & 1 & 0 & 0
\end{array}\right) \\
\bar{E}=\left(\begin{array}{llll}
1 & 0 & 0 & 0 \\
0 & 0 & 0 & 0 \\
0 & 0 & 1 & 0 \\
0 & 0 & 0 & 1
\end{array}\right) F=\left(\begin{array}{ll}
1 & 0 \\
0 & 0 \\
0 & 1 \\
0 & 0
\end{array}\right)
\end{gathered}
$$

where matrices $A_{1}, B_{1}, E$ and $C$ were taken from [22] with structured fault matrix $F$.

The system (1) can be rewritten in the form of system (5), where the augmented matrices $E$ and $C$ are:

$$
E=\left(\begin{array}{ll}
\bar{E} & -F \\
0_{2 \times 4} & I_{2}
\end{array}\right) C=\left(\begin{array}{ll}
\bar{C} & 0_{2 \times 2}
\end{array}\right)
$$

It can be verified that

$$
\operatorname{rank}\left(\begin{array}{c}
E \\
C
\end{array}\right)=n+d=6
$$

The matrices $\alpha, \beta, \gamma$ and $\xi$, satisfying (11) and (12) are computed by using the Singular Value Decomposition property of $(E C)^{T}$ :

$$
\begin{aligned}
& \alpha=\left(\begin{array}{cccccc}
0.333 & 0 & 0 & 0 & 0.333 & 0 \\
0 & 0 & 0 & 0 & 0 & 0 \\
0 & 0 & 1 & 0 & 0 & 1 \\
0 & 0 & 0 & 1 & 0 & 0 \\
-0.333 & 0 & 0 & 0 & 0.667 & 0 \\
0 & 0 & 0 & 0 & 0 & 1
\end{array}\right) \quad \beta=\left(\begin{array}{cc}
0.667 & 0 \\
0 & 1 \\
0 & 0 \\
0 & 0 \\
0.333 & 0 \\
0 & 0
\end{array}\right) \\
& \gamma=\left(\begin{array}{rrrrrr}
-0.471 & 0.577 & 0 & 0 & -0.471 & 0 \\
0.333 & 0.817 & 0 & 0 & 0.333 & 0
\end{array}\right) \xi=\left(\begin{array}{rrr}
0.471 & 0 \\
-0.333 & 0
\end{array}\right)
\end{aligned}
$$

At this point, it is worth to note that the pair $\left(\alpha A_{i},\left(\begin{array}{c}\gamma A_{i} \\ C\end{array}\right)\right)$ is detectable $\forall i=1, \ldots, 2$. An observer of the form (13) can be designed. The disks to place the poles of the matrices $N_{i}$ are $D(0.2,0.8)$ for $i=1,2$. 
Following the procedure described in Section 3, the following matrices are obtained:

$$
\begin{aligned}
& P_{1}=\left(\begin{array}{cccccc}
1.0895 & 0 & 0 & 0 & 0 & 0 \\
0 & 1.0895 & 0 & 0 & 0 & 0 \\
0 & 0 & 0.6681 & 0.3240 & -0.0135 & -1.0236 \\
0 & 0 & 0.3240 & 6.6398 & 0.0825 & 1.1574 \\
0 & 0 & -0.0135 & 0.0825 & 1.0909 & 0.0296 \\
0 & 0 & -1.0236 & 1.1574 & 0.0296 & 2.2746
\end{array}\right) \\
& P_{2}=\left(\begin{array}{cccccc}
1.0895 & 0 & 0 & 0 & 0 & 0 \\
0 & 1.0895 & 0 & 0 & 0 & 0 \\
0 & 0 & 0.6943 & 0.5733 & 0.0165 & -0.9729 \\
0 & 0 & 0.5733 & 5.9887 & 0.0929 & 0.4898 \\
0 & 0 & 0.0165 & 0.0929 & 1.0882 & 0.0002 \\
0 & 0 & -0.9729 & 0.4898 & 0.0002 & 2.0350
\end{array}\right) \\
& J_{1}=\left(\begin{array}{cccccc}
0.6129 & 0 & 0 & 0 & 0 & 0 \\
0 & 0.6129 & 0 & 0 & 0 & 0 \\
0 & 0 & 0.3758 & 0.1823 & -0.0076 & -0.5757 \\
0 & 0 & 0.1823 & 3.7349 & 0.0464 & 0.6510 \\
0 & 0 & -0.0076 & 0.0464 & 0.6136 & 0.0167 \\
0 & 0 & -0.5757 & 0.6510 & 0.0167 & 1.2795
\end{array}\right) \\
& J_{2}=\left(\begin{array}{cccccc}
0.6129 & 0 & 0 & 0 & 0 & 0 \\
0 & 0.6129 & 0 & 0 & 0 & 0 \\
0 & 0 & 0.3905 & 0.3225 & 0.0093 & -0.5473 \\
0 & 0 & 0.3225 & 3.3686 & 0.0523 & 0.2755 \\
0 & 0 & 0.0093 & 0.0523 & 0.6121 & 0.0001 \\
0 & 0 & -0.5473 & 0.2755 & 0.0001 & 1.1447
\end{array}\right) \\
& K=\left(\begin{array}{cc}
0.4714 & -0.3333 \\
00 & \\
5.4512 & 7.7220 \\
-2.5181 & -3.5357 \\
1.1644 & -0.0398 \\
2.7433 & 3.8936
\end{array}\right) \quad L_{21}=\left(\begin{array}{rr}
-0.1000 & 0 \\
-0 & -0.1000 \\
21.9574 & 19.2470 \\
-9.2008 & -8.3224 \\
-0.3503 & 0.1037 \\
10.8547 & 9.6179
\end{array}\right) \quad L_{22}=\left(\begin{array}{rr}
-0.1000 & 0 \\
-0 & -0.1000 \\
14.5827 & 19.0106 \\
-6.5026 & -8.6696 \\
0.0642 & 0.0259 \\
7.1491 & 9.5325
\end{array}\right)
\end{aligned}
$$

By using these gain matrices the following eigenvalues of $N_{i}$ are obtained:

$$
\operatorname{eig}\left(N_{1}\right)=\{0.8499,0.3747,0.1677,0.1076,0.1,0.1\}
$$

and

$$
\operatorname{eig}\left(N_{2}\right)=\{0.6422 \pm 0.0401 i,-0.0983,0.1008,0.1,0.1\}
$$

Finally, matrices $G(\rho), L_{1}(\rho)$ and $N(\rho)$ are computed by means of Eqs. (18)-(20).

The effectiveness of the proposed observer scheme is illustrated by the system studied in open-loop. In this simulation, the time-varying parameter $\rho(k)$ is the measured input, and it is shown in Fig. 1. The weighting functions $\varepsilon_{i}(\rho(k)) i=1,2$ of the LPV system (1) are shown 


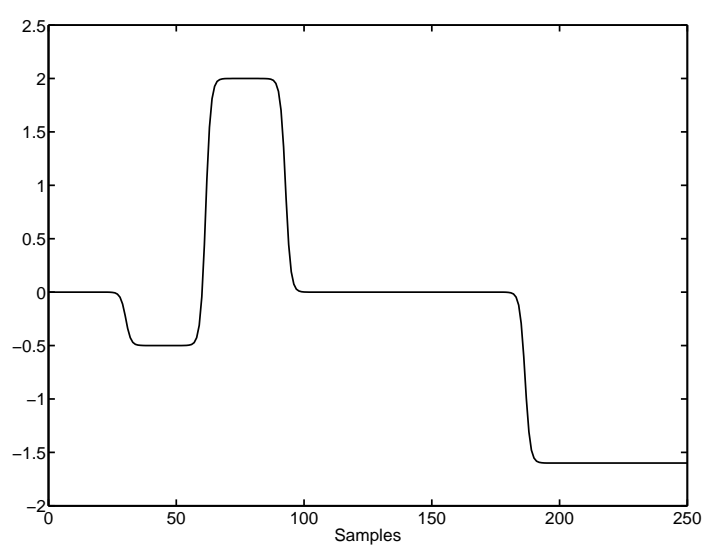

Figure 1. Dynamic behaviour of the input

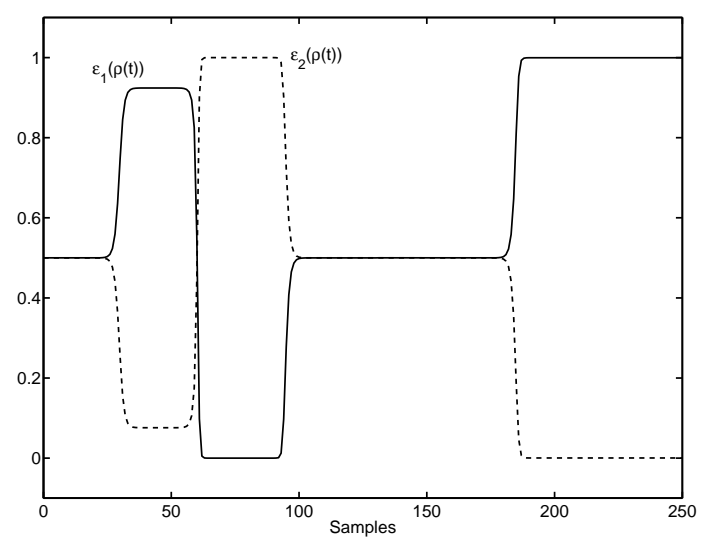

Figure 2. Dynamic behaviour of the weighting functions $\varepsilon_{1}$ (solid line) and $\varepsilon_{2}$ (dashed line)

in Fig. 2. The time evolution of these functions are in accordance with (2). It can be seen that the system is evaluated over the entire operating envelope. Fault-free dynamic behaviour of the outputs is shown in Fig. 3. It can be noted that a gaussian noise is added to the measured outputs.

Two severe faults were simulated. The first fault occurs at the sampled time $t_{k}=50$ and the second fault occurs at $t_{k}=130$. The faults acts as two unknown inputs to the system, affecting the dynamics of the outputs and the states (see Figs. 4-5). The state observer tracks well both, the system states, despite the variations on the input (and consequently the changing operating points), the noisy measurements (see Fig. 4), and the faults. In Fig. 6, it can be appreciated 

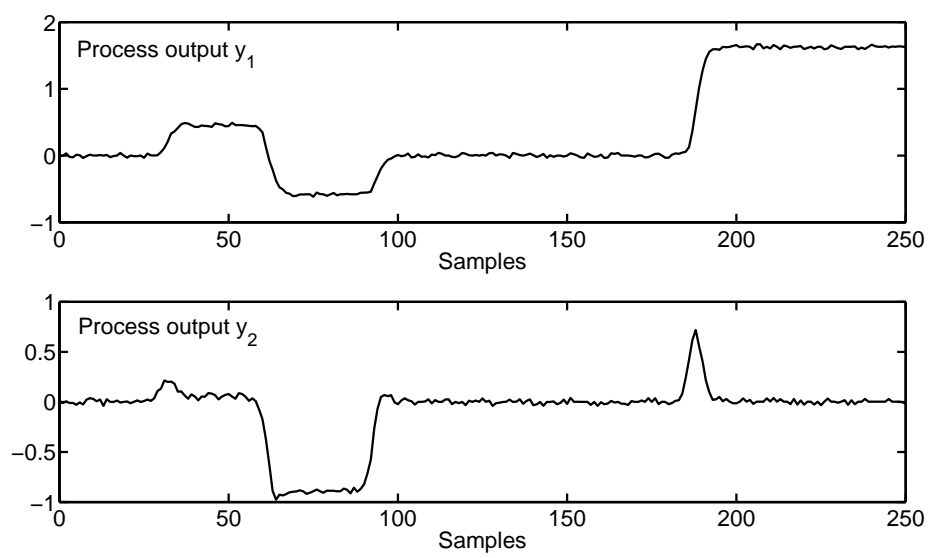

Figure 3. Fault-free dynamic behaviour of the outputs $y_{1}$ and $y_{2}$
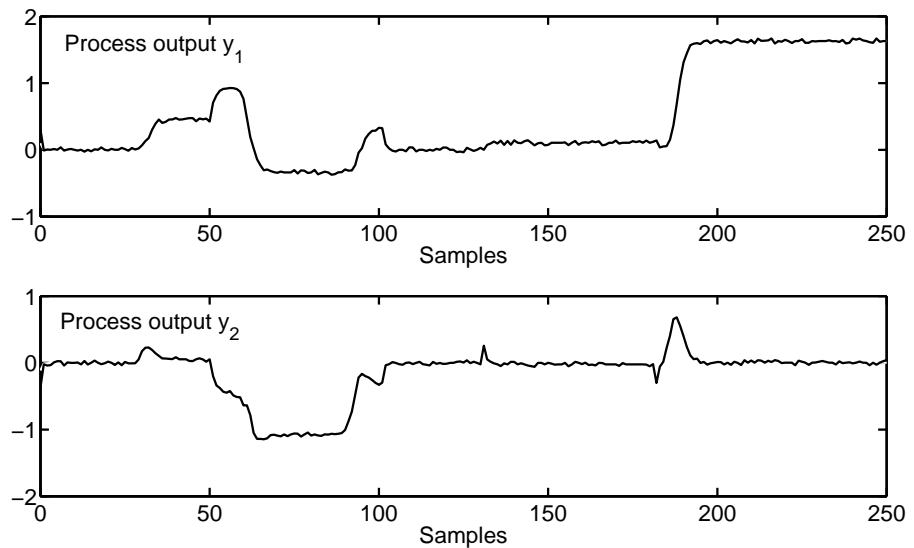

Figure 4. Faulty dynamic behaviour of the outputs $y_{1}$ and $y_{2}$

that the observer detects these faults.

As expected, the observer is noise sensitive. However, these simulation results show that the observer is very effective in detecting and isolating the fault over the entire operating envelope. The estimation of the faults should be used to generate the residuals which can be evaluated through classical statistical methods with the purpose of generate alarms. The fault diagnosis scheme constructed in this way is able to detect, to isolate and to estimate the fault and it represents an efficient tool in the operator's decision. 

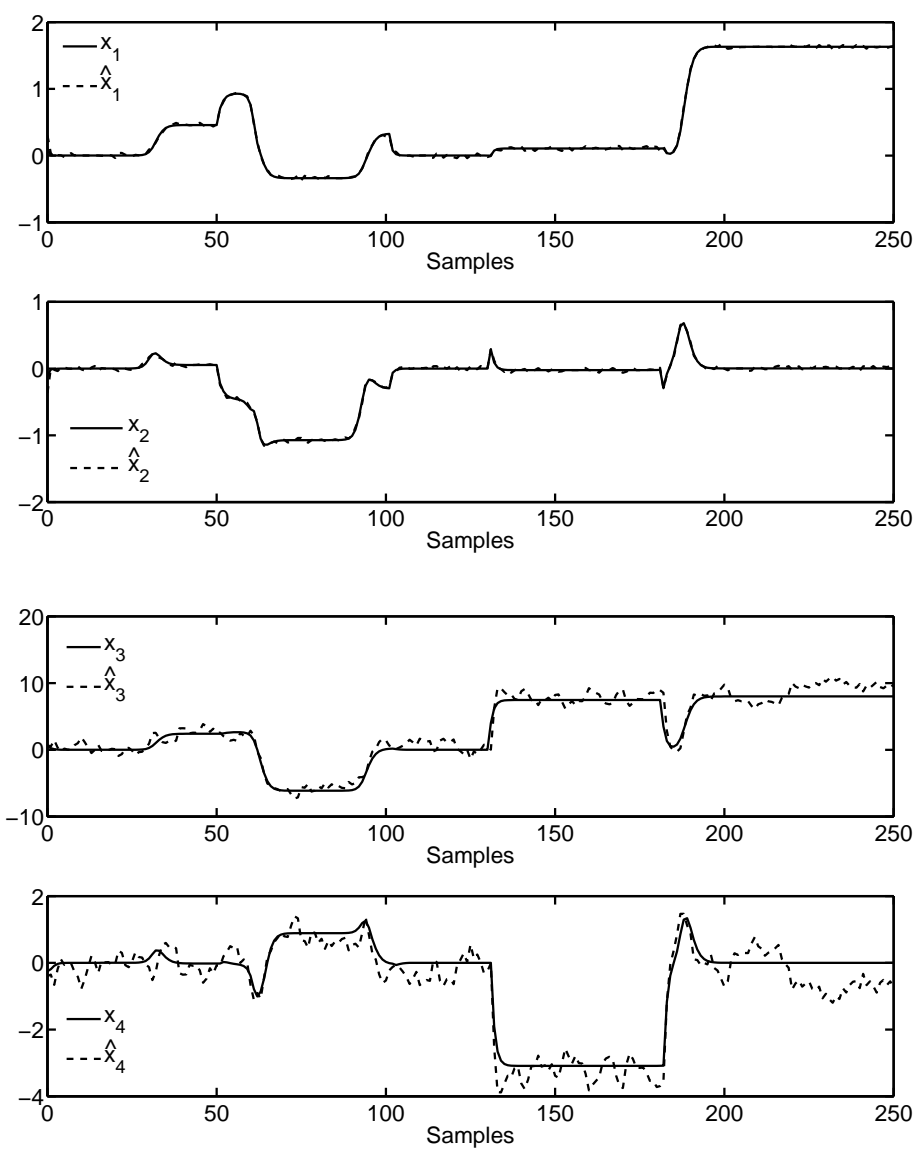

Figure 5. Dynamic behaviour of the states $x_{1}, x_{2}, x_{3}$ and $x_{4}$ and their estimated values $\hat{x}_{1}, \hat{x}_{2}, \hat{x}_{3}$ and $\hat{x}_{4}$

\section{CONCLUSION}

In this paper, a model-based fault diagnosis method for discrete-time D-LPV systems is introduced. For that, an observer that performs fault detection and fault magnitude estimation over the entire operating envelope of the system. The observer synthesis is an extension of the work presented in [25] where an observer for LTI descriptor systems is reported. By taking into account some simple matrix manipulations, this observer can estimate simultaneously both, the states and the fault magnitude vectors, considered as unknown inputs. Sufficient conditions are stated to ensure the existence and the stability of the proposed observer by using a combined Lyapunov analysis based on LMI formulation. An observer gain synthesis has been also proposed to guarantee the efficiency of the model-based fault diagnosis technique. The proposed method is evaluated successfully via numerical simulations. 

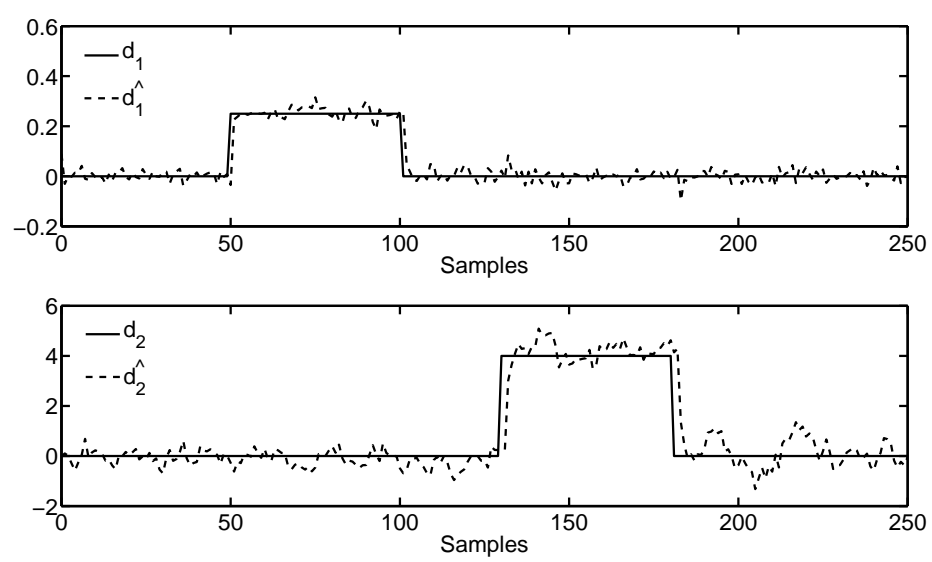

Figure 6. Dynamic behaviour of the faults $d_{1}$ and $d_{2}$ and their estimated values $\hat{d}_{1}$ and $\hat{d}_{2}$

\section{ACKNOWLEDGEMENTS}

The authors would like to thank Prof. Mohamed DAROUACH for most fruitful discussions and Dr. Samir ABERKANE for their recommendations on LMIs. We also thank to PROMEP, Mexico for financial support to Dr. Astorga-Zaragoza during his postdoctoral stage to Centre de Recherche en Automatique de Nancy, France, in 2009. Moreover, the authors would like to thank the anonymous reviewers for their very helpful comments

\section{REFERENCES}

1. Luenberger DG. Dynamic equations in descriptor form. IEEE Transactions on Automatic Control 1977; AC-22(3):312-321.

2. Dai L. Singular control systems. In Lecture Notes in Control and Information Sciences, Thoma M, Wyner A (eds). Springer-Verlag: Berlin, 1989; 118.

3. Ding S.X. Model-based fault diagnosis techniques - Design schemes, algorithms, and tools. In SpringerVerlag: Berlin, 2008; .

4. Müller PC. Stability and optimal control of nonlinear descriptor systems: a survey. Applied Mathematics and Computer Science 1998; 8(2):269-286.

5. Sun SL, Ma J, Lv N. Optimal and self-tuning fusion Kalman filters for discrete-time stochastic singular systems. International Journal of Adaptive Control and Signal Processing 2008; 22(10):932-948.

6. Ishihara JY, Terra MH, Espinoza BM. $H_{\infty}$ filtering for regular discrete-time descrptor systems. Automatica 2009; 45(7):1743-1748.

7. Darouach. $H_{\infty}$ unbiased filtering for linear descrptor systems via LMI. IEEE Transactions on Automatic Control 2009; 54(8):1966-1972.

8. Frisk E, Krysander M, Åslund J. Sensor placement for fault isolation in linear differential-algebraic systems. Automatica 2009; 45(2):364-371.

9. Hu Z, Han Z, Tian Z. Fault detection and diagnosis for singular stochastic systems via B-spline expansions. ISA Transactions 2009; 48(4):519-524.

10. Wei X, del Re L. Gain scheduled $\mathrm{H}_{\infty}$ control for air path systems of diesel engines using LPV techinques. IEEE Transactions on Control Systen Technology 2007; 15(3):406-415.

11. Grenaille S, Henry D, Zolghadri A. A method for designing fault diagnosis filters for LPV polytopic systems. Journal of Control Science and Engineering 2008; 2008:1-11. 
12. Marcos A, Ganguli S, Balas G. An application of $\mathrm{H}_{\infty}$ fault detection and isolation to a transport aircraft. Control Engineering Practice 2005; 13(1):105-119.

13. Armeni S, Casavola A, Mosca E. Robust fault detection and isolation for LPV systems under a sensitivity constraint. International Journal of Adaptive Control and Signal Processing 2009; 23(1):55-72.

14. Abdalla MO, Nobrega G, Grigoriadis KM. Fault detection and isolation filter design for linear parameter varying systems. Proceedings of the American Control Conference, Arlington, VA, USA, June 2001; 38903895 .

15. Balas G, Bokor J, Szabó Z. Failure detection for LPV systems - a geometric approach. Proceedings of the American Control Conference, Anchorage, AK, USA, May 2002; 4421-4426.

16. Bokor J, Balas G. Detection filter design for LPV systems - a geometric approach. Automatica 2004; 40(3):511-518

17. Bara GI, Daafouz J, Kratz F, Ragot J. Parameter-dependent state observer design for affine LPV systems. International Journal of Control 2001; 74(16):1601-1611.

18. Millerioux G, Rosier L, Bloch G, Daafouz J. Bounded state reconstruction error for LPV systems with estimated parameters. IEEE Transactions on Automatic Control 2004; 49(8):1385-1389.

19. Casavola A, Famularo D, Franzè G, Sorbara M. A fault detection, filter-design method for linear parametervarying systems. Proceedings of the Institution of Mechanical Engineers, PArt I: Journal of Systems and Control 2007; 221(6):865-873.

20. Henry D, Zolghadri A. Robust fault diagnosis in uncertain linear parameter-varying sistems. Proceedings of the IEEE International Conference on Systems, Man and Cybernetics, The Hague, NL, October 2004; $5165-5170$.

21. Masubuchi I, Akiyama T, Saeki M. Synthesis of output feedback gain-scheduling controllers based on descriptor LPV system representation. Proceedings of the IEEE Conference on Decision and Control, Maui, Hawaii, USA, December 2003; 6115-6120.

22. Chadli M, Darouach M, Daafouz J. Static output stabilisation of singular LPV systems: LMI formulation. Proceedings of the IEEE Conference on Decision and Control, Cancun, Mexico, December 2008; 47934796.

23. Masubuchi I, Suzuki A. Gain-scheduled controller synthesis based on new LMIs for dissipativity of descriptor LPV systems. Proceedings of the 17th IFAC World Congress, Seoul, Korea, July 2008; 99939998.

24. Bouali A, Yagoubi M, Chevrel P. $\mathrm{H}_{2}$ gain scheduled observer based controllers for rational LPV systems. Proceedings of the IEEE International Conference on Control, Automation, Robotics and Vision, Hanoi, Vietnam, December 2008; 1811-1816.

25. Darouach M, Boutayeb M. Design of observers for descriptor systems. IEEE Transactions on Automatic Control 1995; 40(7):1323-1327.

26. Daafouz J, Bernussou J. Parameter dependent Lyapunov functions for discrete time systems with time varying parametric uncertainties. Systems \& Control Letters 2001; 43(5):355-359.

27. Gao H, Wang Z, Wang C. Improved $H_{\infty}$ control of discrete-time fuzzy systems: a cone complementary linearization approach. Information Sciences 2005; 175(1-2):57-77.

28. El-Ghaoui L, Oustry F, AitRami M. A cone complementarity linearization algorithm for static outputfeedback and related problems. IEEE Transactions on Automatic Control 1997; 42(8):1171-1176.

29. Kim BS, Han HS, Lee JG. Pole placement of uncertain discrete systems in the smallest disk by state feedback. Proceedings of the 35th Conference on Decision and Control, Kobe, Japan, December 1996; 4558-4563. 